Old Dominion University

ODU Digital Commons

Physics Faculty Publications

Physics

4-2008

\title{
Investigation of Loading of Pulsed and Continuous-Wave Optical Dipole Force Traps
}

\author{
M. Shiddiq \\ Old Dominion University \\ E. M. Ahmed \\ Old Dominion University \\ M. D. Havey \\ Old Dominion University, mhavey@odu.edu \\ C. I. Sukenik \\ Old Dominion University, csukenik@odu.edu
}

Follow this and additional works at: https://digitalcommons.odu.edu/physics_fac_pubs

Part of the Atomic, Molecular and Optical Physics Commons

\section{Repository Citation}

Shiddiq, M.; Ahmed, E. M.; Havey, M. D.; and Sukenik, C. I., "Investigation of Loading of Pulsed and Continuous-Wave Optical Dipole Force Traps" (2008). Physics Faculty Publications. 4.

https://digitalcommons.odu.edu/physics_fac_pubs/4

\section{Original Publication Citation}

Shiddiq, M., Ahmed, E.M., Havey, M.D., \& Sukenik, C.I. (2008). Investigation of loading of pulsed and continuous-wave optical dipole force traps. Physical Review A, 77(4), 045401. doi: 10.1103/PhysRevA.77.045401

This Article is brought to you for free and open access by the Physics at ODU Digital Commons. It has been accepted for inclusion in Physics Faculty Publications by an authorized administrator of ODU Digital Commons. For more information, please contact digitalcommons@odu.edu. 


\title{
Investigation of loading of pulsed and continuous-wave optical dipole force traps
}

\author{
M. Shiddiq, E. M. Ahmed, M. D. Havey, and C. I. Sukenik \\ Old Dominion University, Department of Physics, Norfolk, Virginia 23529, USA
}

(Received 20 September 2007; published 2 April 2008)

\begin{abstract}
We have investigated the behavior of an optical dipole force trap realized using a mode-locked Nd:YAG laser and have compared performance to a continuous-wave (cw) trap built using the same laser but running in a cw mode. The traps are used to confine ultracold ${ }^{85} \mathrm{Rb}$ atoms which are loaded from a magneto-optical trap (MOT). In most respects, the two traps behave similarly over a wide range of laser parameters provided that the average potential well depth is the same; however, there is a notable difference in the dipole trap loading efficiency dependence on the detuning of the MOT trap laser frequency during the loading stage.
\end{abstract}

DOI: 10.1103/PhysRevA.77.045401

PACS number(s): 37.10.De, 37.10.Gh, 37.10.Vz

The magneto-optical trap (MOT) is widely used in atomic and molecular physics experiments to provide samples of ultracold atoms. Rescattering of the near-resonant light used to construct the MOT, however, limits the densities achievable in the trap. When higher densities are required, an optical dipole force trap is frequently used. Unlike a MOT, an optical dipole force trap can also be used to confine molecules [1]. If the trap laser is very far detuned from an atomic resonance, the trap is referred to as a "far-off-resonance trap" or "FORT." Because the optical dipole force is nearly conservative, pre-cooled atoms must be loaded into the trap. Trap loading is usually done by transferring atoms from a MOT. The dynamics of such a transfer are complicated and have been investigated by several groups [2-4].

Although to date most dipole force traps use a continuous-wave (cw) laser, pulsed laser FORTs have also been demonstrated. There are a number of reasons why using a pulsed FORT is attractive for ultracold experiments. Expanding the available spectrum of light for trapping will expand the number of atomic species which can be confined. The high intensities found in short pulse lasers enable nonlinear frequency conversion to be used to produce light in the blue end of the frequency spectrum where cw lasers sources are difficult to obtain. Such light could be used to create blue-detuned traps for a number of species. Pulsed traps are also interesting for metrology applications because the resonant frequency shift for an atom in a periodic perturbation is different from that of the cw case [5]. In addition, very high average power lasers, such as the free-electron laser at Thomas Jefferson National Laboratory (JLab) could be used to make energetically deep or spatially large optical traps for cold atoms and molecules. The JLab free-electron laser is a subpicosecond laser with a pulse repetition rate of 5-80 $\mathrm{MHz}$. If pulsed lasers are to be applied as above, then understanding the trapping dynamics of such a system is critical.

A pulsed FORT using a mode-locked laser was first reported in Ref. [6], using both a Ti:sapphire and a Nd: $\mathrm{YVO}_{4}$ laser. There, comparable performance between $\mathrm{cw}$ and pulsed operation of each trap was reported. The expected loading behavior of a pulsed and cw trap is discussed in a previous publication [7] in which we reported on studies with a pulsed (Nd:YAG, $\sim 100 \mathrm{ps}, 80 \mathrm{MHz}$ repetition rate) FORT for ${ }^{85} \mathrm{Rb}$ atoms. Provided that the pulse repetition rate does not drive parametric oscillations in the trap, for large detunings one would expect the same behavior from each trap if the average power is the same. We found that in most respects (but not all), the pulsed and cw traps are quite similar.

In this Brief Report, we present results and analysis of our study of the loading behavior of a pulsed FORT. Loading rates and loss rates of atoms in the trap have been studied over a range of parameters which include power of the FORT laser beam (the trap well depth), detuning of the MOT trapping laser during loading, hyperfine repump laser intensity during loading, and MOT trapping laser intensity. We compare pulsed FORT operation to a $\mathrm{cw}$ FORT with the same average power.

Details of the apparatus, shown schematically in Fig. 1, can be found in Ref. [7]. Briefly, a standard ${ }^{85} \mathrm{Rb}$ MOT, with $\sim 1.5 \times 10^{7}$ atoms is used as the source of cold atoms. An injection-locked diode laser is used for the trapping beams and a separate laser is used for the hyperfine repumper (HFR). Using a separate HFR laser enables precise intensity and frequency control, which is required for efficient loading. Atoms are transferred to the FORT by overlapping a $\mathrm{Nd}$ :YAG laser beam, focused to a calculated waist radius of $\sim 16 \mu \mathrm{m}$, with the $\mathrm{Rb}$ cloud, following the timing protocol discussed in Ref. [7]. The number of atoms in the FORT is determined by measuring the fluorescence of atoms in an optical molasses phase following the FORT loading phase

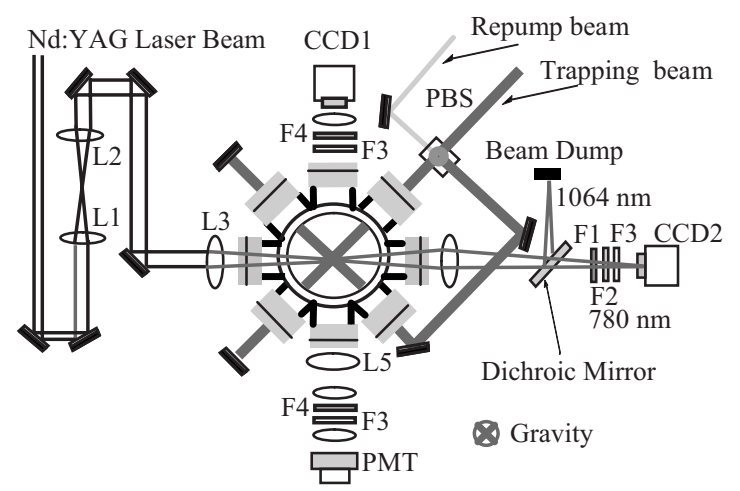

FIG. 1. The experimental setup for a $\mathrm{cw}$ and a pulsed FORT. $L 1=100 \mathrm{~mm}$ and $L 2=200 \mathrm{~mm}$. $F 1=$ neutral density filter, $F 2$ $=$ colored glass filter, $F 3=$ interference filter, and $F 4=$ short pass filter. $\mathrm{PBS}=$ polarizing beam splitter, $\mathrm{PMT}=$ photomultiplier, and $\mathrm{CCD}=$ charge coupled device camera. 


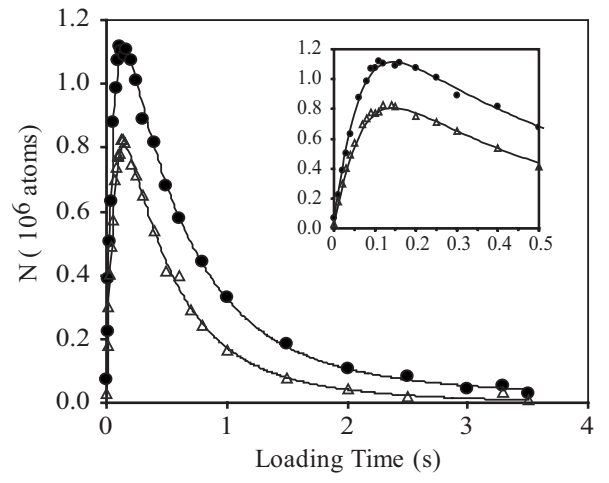

FIG. 2. Number of atoms as a function of loading time in a cw $(\triangle)$ and a pulsed $(\triangle)$ FORT for $7 \mathrm{~W}$ of FORT laser beam power, $-8 \Gamma$ MOT laser detuning, $18.0 \mu \mathrm{W} / \mathrm{cm}^{2}$ repump laser intensity, and $23.1 \mathrm{~mW} / \mathrm{cm}^{2}$ trap laser intensity.

and holding period (during the holding period, only the FORT light is present). For probing, the MOT and hyperfine repumper laser beams are on but no magnetic field gradient is present.

Following Ref. [2] we use a rate equation approach to characterize the number of atoms loaded into the FORT as a function of time:

$$
\frac{d N}{d t}=R_{0} e^{\left(-\gamma_{\mathrm{MOT}} t\right)}-\Gamma_{L} N-\beta_{L}^{\prime} N^{2} .
$$

The number of atoms remaining in the FORT once loading is terminated is given by the solution to

$$
\frac{d N}{d t}=-\Gamma_{H} N-\beta_{H}^{\prime} N^{2} .
$$

Here $R_{0}$ is the loading rate, $\gamma_{\text {MOT }}$ characterizes the change to the MOT dynamics during the FORT loading stage, $\Gamma_{i}$ is the loss rate due to background gas collisions, and $\beta_{i}^{\prime}$ is the loss rate from two-body collisions of trapped atoms. The indices $L$ and $H$ are used to differentiate loss rates during loading from those during holding which may be different. The prime on $\beta^{\prime}$ is to differentiate number-dependent loss rates from density-dependent loss rates. The dynamics of the transfer process from a MOT to a cw and pulsed FORT are explored by measuring the number of atoms in the FORT as a function of time for different laser parameters and extracting the coefficients in the above equations by fitting the experimental data to the solutions of Eq. (1) or Eq. (2).

We have investigated the effect of changes in the loading time, the power of the FORT laser, the intensity of the hyperfine repump laser, the intensity of the trap laser, and the detuning of the trap laser frequency during FORT loading. Combinations of these parameters provide a rather large phase space in which to navigate. We have tried to cover as much of the space as needed to observe the essential behavior of the trap. Figure 2 shows typical loading curves of the $\mathrm{cw}$ and pulsed FORT. Here the FORT laser power incident on the chamber is $7 \mathrm{~W}$. All FORT powers quoted here refer to the measured incident power. The chamber windows are not antireflection coated, so that actual FORT power at the

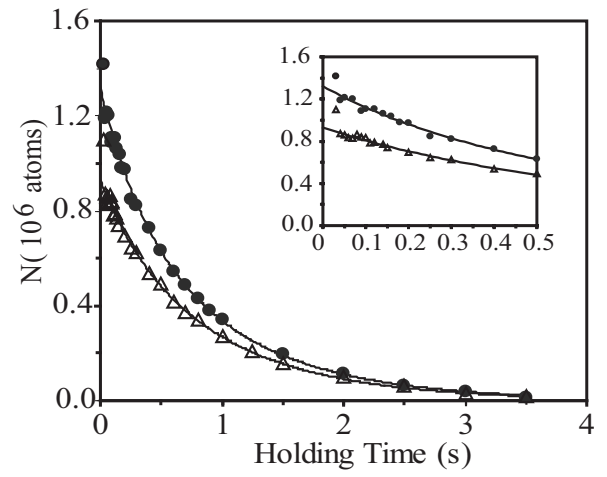

FIG. 3. Number of atoms vs holding time in a cw (0) and a pulsed $(\triangle)$ FORT for $7 \mathrm{~W}$ of FORT laser beam power, $-8 \Gamma$ MOT laser detuning, $18.0 \mu \mathrm{W} / \mathrm{cm}^{2}$ repump laser intensity, and $23.1 \mathrm{~mW} / \mathrm{cm}^{2}$ trap laser intensity.

location of the atoms is reduced by $\sim 8 \%$ from the measured incident power. For a Nd:YAG beam waist radius $\sim 16 \mu \mathrm{m}$, this yields a FORT trap depth of $\sim 2.1 \mathrm{mK}$. During the loading phase, the MOT laser detuning is set to $-8 \Gamma$ ( $\Gamma$ is the natural linewidth of $5.9 \mathrm{MHz}), 18.0 \mu \mathrm{W} / \mathrm{cm}^{2}$ HFR laser intensity and $23.1 \mathrm{~mW} / \mathrm{cm}^{2}$ trap laser intensity, corresponding to the optimum values for FORT loading. The maximum number of atoms transferred from the MOT to the FORT for both pulsed and cw traps occurs at $\sim 110 \mathrm{~ms}$ loading time.

There are $\sim 1.5 \times 10^{7}$ ultracold $\mathrm{Rb}$ atoms in the MOT. We have observed that the pulsed FORT systematically loads fewer atoms than the cw FORT under otherwise "identical" conditions. At $7 \mathrm{~W}$ FORT laser power, about $8 \%$ of the atoms are loaded successfully into the cw FORT and about 5\% into the pulsed FORT. At $4 \mathrm{~W}$ FORT laser power, 5.5 and $4 \%$ of the atoms are loaded into the $\mathrm{cw}$ and pulsed FORT, respectively. The FORT power dependence data suggests that the effective trap depth for pulsed and cw FORTs differs up to $40 \%$ even when the average incident power is the same. We are investigating whether it is spatial mode imperfections of the mode-locked laser or other effects that are responsible, but the data is not conclusive at this time. We therefore focus our study on dependencies of parameters when comparing the pulsed and cw traps.

After loading is optimized the MOT lasers and $B$-field gradient are extinguished, leaving only the FORT laser to confine the atoms. With loading terminated, trap atom number begins to decrease as atoms are lost due to heating and collisions. Figure 3 shows typical holding curves in both a $\mathrm{cW}$ and a pulsed FORT for $7 \mathrm{~W}$ average incident FORT laser beam power. We have recorded a set of holding curves taken at FORT powers of 4-7 $\mathrm{W}$ to extract $\Gamma_{H}$ and $\beta_{H}^{\prime}$. The curves are analyzed for times greater than $100 \mathrm{~ms}$ to allow untrapped atoms to leave the FORT volume and so as not to include any evaporative cooling effects. We did not find any difference between pulsed and $\mathrm{cw}$ values of these parameters. The average $\Gamma_{H}$ is $\sim 1 / \mathrm{s}$ thereby making the lifetime of the trap $\left(1 / \Gamma_{H}\right) \sim 1 \mathrm{~s}$, due primarily to the vacuum chamber background pressure. The loss rate due to two body collisions $\beta_{H}^{\prime}$ at the average FORT laser power of $7 \mathrm{~W}$ is $4.2 \pm 0.7 \times 10^{-7}{\text { (atoms s})^{-1}}$ for the $\mathrm{cW}$ FORT and $4.6 \pm 1.0 \times 10^{-7}$ (atoms s) $^{-1}$ for the pulsed FORT. As 


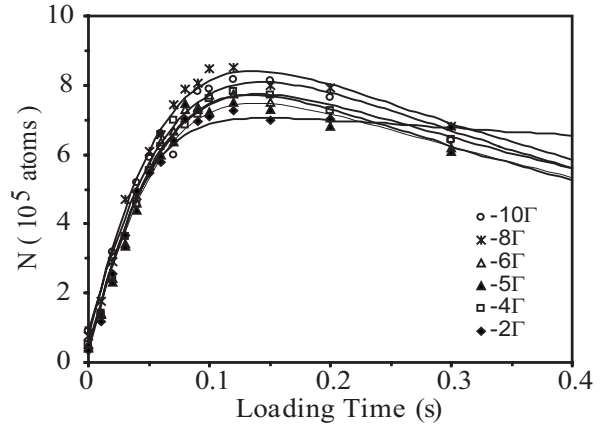

FIG. 4. Number of atoms as a function of loading time in a cw FORT for different trap laser detunings at $7 \mathrm{~W}$ FORT laser power, $13.1 \mu \mathrm{W} / \mathrm{cm}^{2}$ repump laser intensity, and $23.1 \mathrm{~mW} / \mathrm{cm}^{2}$ trap laser intensity.

expected, $\beta_{H}^{\prime}$ decreases as the potential depth increases.

An analysis of the loading curves, shows a similar loading rate of $R_{0} \sim 1.8 \times 10^{7}$ atoms s${ }^{-1}$ and a loss rate of $\beta_{L}^{\prime} \sim 1 \times 10^{-5}$ (atoms s) ${ }^{-1}$ for the two traps. In reality, the loading rate for pulsed loading is $\sim 25 \%$ less than that for $\mathrm{cw}$ and the loss rate is $\sim 25 \%$ larger. The power dependence for both traps shows a near constant $R_{0}$, but a threefold increase in $\beta_{L}^{\prime}$ as the FORT power is decreased from 7 to $4 \mathrm{~W}$. Although lowering the MOT intensity during FORT loading could aid in sub-Doppler cooling, we did not find an enhancement here in the FORT loading efficiency when the MOT laser intensity was lowered. We observed that the loading of both traps increased linearly with MOT power up to an intensity of $12 \mathrm{~mW} / \mathrm{cm}^{2}$ and then saturated. $R_{0}$ was essentially constant with MOT intensity and $\beta_{L}^{\prime}$ decreased slightly and then leveled off as a function of $I_{\mathrm{MOT}}$. In the FORT loading stage, the trap laser detuning has an important role in getting the largest percentage transfer of ultracold atoms in the MOT to the FORT [2]. As the FORT only confines - but does not cool—atoms, it is important to maintain the cooling process as atoms are loaded into the FORT. While atoms in the MOT-FORT-overlap region are being cooled, atoms outside of the FORT volume must also be transported to the FORT region to be loaded. The greatest overall loading efficiency occurs by maximizing the cooling of atoms as they load into the FORT while reducing any

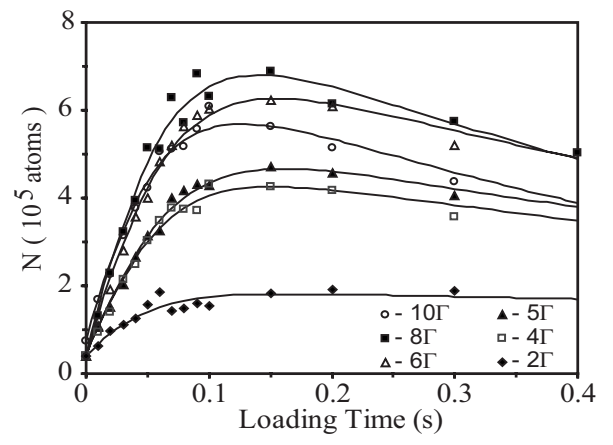

FIG. 5. Number of atoms as a function of loading time in a pulsed FORT for different trap laser detunings at $7 \mathrm{~W}$ FORT laser power, $13.1 \mu \mathrm{W} / \mathrm{cm}^{2}$ repump laser intensity, and $23.1 \mathrm{~mW} / \mathrm{cm}^{2}$ trap laser intensity.

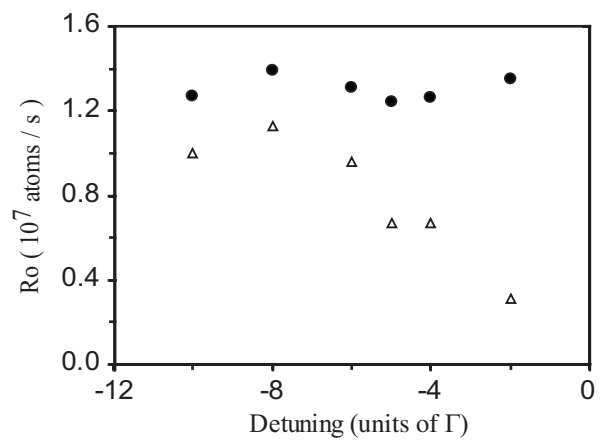

FIG. 6. Loading rates vs trap laser detuning in a cw $(0)$ and a pulsed $(\triangle)$ FORT for $7 \mathrm{~W}$ FORT laser power, $13.2 \mu \mathrm{W} / \mathrm{cm}^{2}$ repump laser intensity, and $23.1 \mathrm{~mW} / \mathrm{cm}^{2}$ trap laser intensity.

negative impact to MOT atom transport that a detuning change brings.

Figures 4 and 5 display loading curves at different MOT trap laser detunings during loading for $\mathrm{cw}$ and pulsed operation, respectively. Both FORTs have maximum loading at $-8 \Gamma$ detuning. Closer to resonance, the $\mathrm{cw}$ FORT still loads approximately the same until the detuning reaches $-2 \Gamma$, however, the pulsed FORT shows a degradation in FORT loading starting at $-5 \Gamma$ and loads very poorly as the MOT detuning approaches resonance. In Figs. 6 and 7, $R_{0}$ and $\beta_{L}^{\prime}$ are shown as a function of detuning. Our $\mathrm{cw}$ results are in qualitative agreement with those of Ref. [8]. The poor performance of the pulsed FORT for small detuning arises from a decrease in $R_{0}$ accompanied by an increase in $\beta_{L}^{\prime}$. Simulation of the loading curves shows that a reduction of $R_{0}$ by a factor of 4 from its peak value at $-8 \Gamma$, results in a $58 \%$ reduction in the number of atoms loaded, while increasing $\beta_{L}^{\prime}$ by the same factor yields a $43 \%$ reduction. We also find that $\gamma_{\mathrm{MOT}}$ decreases smoothly for the pulsed FORT as the detuning is reduced, whereas it tends to remain nearly constant for $\mathrm{cW}$ operation until the detuning approaches $-2 \Gamma$. $\gamma_{\mathrm{MOT}}$ can be measured approximately by observing the MOT fluorescence decay at a time $T_{D}$ before FORT loading would begin [8] or by extracting it from a fit to the loading curves.

We have investigated other combinations of parameters, including the detuning dependence discussed above at different FORT powers and hyperfine repump laser intensity. We

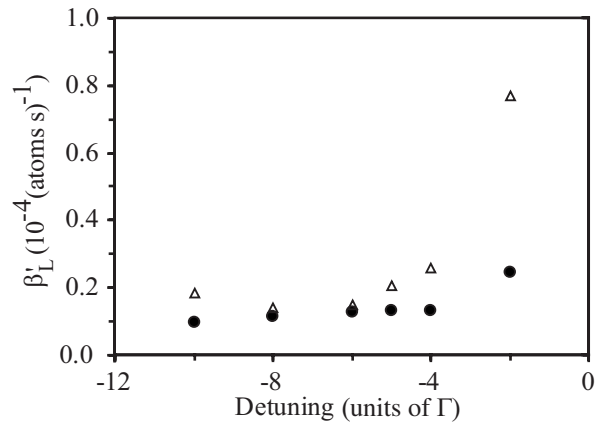

FIG. 7. Loss rates vs trap laser detuning in a cw $(0)$ and a pulsed $(\triangle)$ FORT for $7 \mathrm{~W}$ FORT laser power $13.2 \mu \mathrm{W} / \mathrm{cm}^{2}$ repump laser intensity and $23.1 \mathrm{~mW} / \mathrm{cm}^{2}$ trap laser intensity. 
observed that the shape of the detuning dependence curves for a cw FORT at low FORT power resembles the curve shape for the pulsed FORT at all powers studied. At optimal trap loading of $-8 \Gamma$ detuning there was little difference between pulsed and cw dependence on $I_{R}$. After the optimal value of repump laser intensity is reached, $R_{0}$ decreased and $\beta_{L}^{\prime}$ rose with both traps exhibiting a similar dependence on $I_{R}$. At $-3 \Gamma$, however, $R_{0}$ for the pulsed FORT is considerably smaller than for the cw case at low repump laser intensity, while $\beta_{L}^{\prime}$ is much larger than the cw case at higher $I_{R}$.

Having observed a difference in the loading and loss rates for cw and pulsed traps, our investigation is now focused on understanding the physical mechanism(s) responsible for the observed behavior. Multiple mechanisms seem likely. For example, the detuning dependence data at different powers suggests that the reduced loading rate may arise from a reduction in the cooling of atoms during pulsed FORT loading in near resonant MOT light-perhaps due to conditions which are less favorable for polarization gradient cooling. At the same time, the observed increase in the loss rate may indicate that photoassociative losses are more problematic in the pulsed FORT than its cw counterpart. Future studies will include spectroscopic investigation to determine the resonant frequency of atoms in the FORT [5] as well as detailed temperature measurements of atoms loaded into the FORT. The latter could provide direct evidence of a reduction of cooling efficacy in the pulsed FORT. The experiment was initiated by a desire to investigate whether there was an appreciable difference in the physics of pulsed FORT loading in comparison to $\mathrm{cW}$ FORT loading. Our principal finding is that in most respects, the behavior of the two types of traps is similar; however, there is a difference in the FORT loading efficiency dependence on the detuning of the MOT trap laser frequency during the loading phase. Our interest now is in developing a better understanding of the physical mechanisms responsible for the observed differences.

In conclusion, FORT loading is a complicated dynamical process and many aspects of the physics of FORT loading require future study. In addition to continued studies of the dependence on the detuning, future studies should include the dependence of loading on the polarization of the FORT laser light (circular and elliptical FORT), the dependence of the characteristics of the mode-locked laser (fs vs ps pulses), the dependence on FORT to MOT size ratio, and a study of how the temperature of atoms in the FORT depends on the many loading parameters.

We gratefully acknowledge support from the NSF, Jefferson Laboratory, and Old Dominion University. We thank R. R. Jones and D. Cho for collaborative assistance and J. Sebby-Strabley for discussions on FORT laser alignment techniques.
[1] T. Takekoshi, B. M. Patterson, and R. J. Knize, Phys. Rev. Lett. 81, 5105 (1998).

[2] S. J. M. Kuppens, K. L. Corwin, K. W. Miller, T. E. Chupp, and C. E. Wieman, Phys. Rev. A 62, 013406 (2000).

[3] K. L. Corwin, S. J. M. Kuppens, D. Cho, and C. E. Wieman, Phys. Rev. Lett. 83, 1311 (1999).

[4] K. M. O'Hara, S. R. Granade, M. E. Gehm, and J. E. Thomas, Phys. Rev. A 63, 043403 (2001).

[5] J. M. Choi, G.-N. Kim, D. Cho, and C. I. Sukenik, J. Korean
Phys. Soc. 51, 294 (2007).

[6] R. B. M. Clarke, T. Graf, and E. Riis, Appl. Phys. B: Lasers Opt. 70, 695 (2000).

[7] M. Shiddiq, C. E. Lucas, M. D. Havey, C. I. Sukenik, R. R. Jones, C. Y. Park, J. Y. Kim, and D. Cho, J. Korean Phys. Soc. 47, 207 (2005).

[8] K. L. Corwin, Ph.D. thesis, University of Colorado, 1999 (unpublished). 